\title{
Antimicrobial Susceptibility Pattern of Bacteriuria and Risks of Therapeutic Failure among Pregnant Women in Primary Health Care of Macau, China
}

\author{
Mei-Fong Chou ${ }^{1}$, Chau-Sha Kwok ${ }^{2}$, Chon-Kit Ieong ${ }^{3}$, Sai-Meng Pang ${ }^{2}$ \\ ${ }^{1}$ Department of Areia Preta Health Center, Macau, China \\ ${ }^{2}$ Alameda Dr. Carlos d'Assumpcao, Macau, China \\ ${ }^{3}$ Centro Hospitalar Conde de São Januário, CHCSJ, Macau, China \\ Email: donestella89@gmail.com
}

How to cite this paper: Chou, M.-F., Kwok, C.-S., Ieong, C.-K. and Pang, S.-M. (2019) Antimicrobial Susceptibility Pattern of Bacteriuria and Risks of Therapeutic Failure among Pregnant Women in Primary Health Care of Macau, China. Open Journal of Obstetrics and Gynecology, 9, 1347-1357.

https://doi.org/10.4236/ojog.2019.910130

Received: August 27, 2019

Accepted: October 11, 2019

Published: October 14, 2019

Copyright $\odot 2019$ by author(s) and Scientific Research Publishing Inc. This work is licensed under the Creative Commons Attribution International License (CC BY 4.0).

http://creativecommons.org/licenses/by/4.0/

\begin{abstract}
Both asymptomatic (ASB) and symptomatic bacteriuria during pregnancy increase the risk of adverse fetal or maternal outcomes. Our study aims to determine the prevalence of bacteriuria, the etiological agents with their susceptibility to antimicrobials, and the risk factors for therapeutic failure among pregnant women for antenatal care in the primary health care of Macau, China. Methods: This is a cross-sectional survey involving 5101 antenatal care pregnant women from $1^{\text {st }}$ Jan to $31^{\text {st }}$ Dec., 2017. The mid-stream clean-catch urine specimens were routinely examined microscopically and cultured following the first consultation. Other data were collected from the computerized medical records. Results: The mean age of the pregnant women was $31.4 \pm 5.04$ years. We followed 130 women with positive cultures on first consultation, of whom 35 (26.9\%) were found to have contaminated cultures. 95 pregnant women were confirmed to have UTI. The prevalence of bacteriuria was $1.86 \%(\mathrm{n}=95)$, of which $83(87.4 \%)$ were asymptomatic. ASB was mainly in the younger age-group (20 - 30 years) and the primipara. Of 196 positive cultures, the commonest bacterium was Escherichia coli $(\mathrm{n}=111$ or $56.6 \%, 98.2 \%$ susceptible to nitrofurantoin), followed by Klebsiella pneumonia ( $\mathrm{n}=20$ or $10.2 \%, 90 \%$ susceptible to $2^{\text {nd }}$ generation cephalosporin), and Streptococcus agalactiae ( $\mathrm{n}=16$ or $8.2 \%, 100 \%$ susceptible to nitrofurantoin). After treatment, there were $80(84.2 \%)$ therapeutic successes and 15 (15.8\%) failures. Symptomatic infection and later trimester were significantly associated with therapeutic failure $(P \leq 0.05)$. Conclusions: ASB is common in the young age group and primipara. Nitrofuranton and $2^{\text {nd }}$ generation cepha-
\end{abstract}


losporin are effective antibiotics against UTI in pregnancy. Symptomatic infection and occurrence in later trimester were significantly associated with treatment failure.

\section{Keywords}

Pregnancy, Asymptomatic Bacteriuria (ASB), Symptomatic Bacteriuria (SB), Primary Health Care, Antenatal Care

\section{Introduction}

Pregnant women are more susceptible to urinary tract infections (UTI) due to several anatomical and hormonal changes. UTI is defined as two consecutive aseptically collected midstream urine specimens with the isolation of the same bacterial strain in quantitative counts of $\geq 10^{5}$ colony-forming units (CFU)/ml [1]. Most of these UTI present as asymptomatic bacteriuria (ASB). The prevalence of ASB in pregnant women was about $2 \%-7 \%$ [2]. Both ASB and symptomatic bacteriuria (SB) during pregnancy increase the risk of pyelonephritis and have been associated with adverse pregnancy outcomes, such as preterm births, low birth weight infants, preeclampsia, and perinatal mortality [3] [4].

UTI in pregnancy needs aggressive therapy. Studies reveal that the risk of pyelonephritis can be reduced by $70 \%-80 \%$ if bacteriuria is aggressively eradicated [5]. From a meta-analysis of 14 randomized trials, antibiotic therapy can clear ASB (odds ratio [OR] 0.30, 95\% CI: 0.18 - 0.53) and lower the incidence of pyelonephritis (OR 0.23, 95\% CI: $0.13-0.41$ ), NNT = 6 (95\% CI: $5.0-7.0$ ). Antibiotic therapy will also reduce the incidence of low birth weight infants (RR 0.63 , 95\% CI: $0.45-0.90$; NNT $=23,95 \%$ CI: 15 - 85) [6]. Some studies show that the treatment success depends on the causative bacteria's susceptibility to antimicrobials rather than on the duration of therapy [7]. But others report that treatment failure is present up to $30 \%$ of ASB in a short course of therapy [8]. Furthermore, other factors that are associated with failure to treat bacteriuria are not known in recent studies.

In the absence of local data, this study aimed to determine the prevalence, the etiological bacteria with their susceptibility to antibiotics, and the therapeutic failure rate with its risk factors among pregnant women for antenatal care in the public primary healthcare of Macau, China.

\section{Methods}

\subsection{Study Design}

This is a retrospective cross-sectional study from $1^{\text {st }}$ Jan., 2017 to $31^{\text {st }}$ Dec., 2017. Up to date, there are seven Health Centers and three Health Stations in Macau that provide antenatal care to all women who choose delivery in the public service. High risk pregnancies would be referred to the Obstetrics Department in 
the Centro Hospital ar Conde de São Januário (CHCSJ, the central hospital) for further care. Data were extracted from the computerized medical records of these women. Ethical approval was obtained from the Research Ethics Committee of the Hospital Authority.

\subsection{Data Collection}

At the first consultation of the antenatal clinic, a nurse taught each patient how to collect aseptically a mid-stream urine specimen into a sterile container. The specimen was transported to the Department of Microbiology, Centro Hospital ar Conde de São Januário (CHCSJ), in the following morning.

Urine specimens underwent microscopic examination and culture by Quantitative Culture Plate Method with bio Merieux VITEK 2 Systems for antibiotic susceptibility tests. Bacteriuria was defined as over $10^{5} \mathrm{CFU} / \mathrm{ml}$ growth on culture without leukocytes esterase on microscopy, or $10^{4}-10^{5} \mathrm{CFU} / \mathrm{ml}$ in culture with $>500 \mathrm{WBCs} / \mu$ l leukocytes esterase on microscopy. MSU examination was repeated in all asymptomatic women. If the second specimen showed no bacteria or different flora, the first MSU was regarded as contamination.

A course of antibiotic according to the susceptibility results was given to asymptomatic women with the same bacterium isolated in the repeated MSU culture. The appropriate antibiotics were also prescribed to the women with symptomatic bacteriuria. All treated patients were instructed to provide a MSU for culture and susceptibility test again after treatment. Persistent bacteriuria after a course of antibiotics was regarded as therapeutic failure.

We reviewed the reports of all positive cultures for the etiological bacteria and their susceptibility for antibiotics. We also reviewed for each patient the therapeutic failure rate and analyzed their risk factors. Age, parity, gestational age, and history of UTI, anemia, gestational diabetes mellitus (GDM) were included for analysis. Those patients who were lost to follow-ups, had natural abortion or stillbirth in this pregnancy, or had taken antibiotics in recent two weeks were excluded for assessment in our study.

\subsection{Statistical Analysis}

Data analysis was descriptive and binomial logistic regression was used to assess factors associated with treatment outcome (success/failure). A $P$-value of $\leq 0.05$ was considered statistically significant. We take the definition of prevalence as the total number of individuals in a population who have a disease or health condition at a specific period of time, expressed as a percentage of the population.

\section{Results}

\subsection{Distribution of Recruited Women}

In year 2017, 5101 women visited the antenatal clinics in public primary care. Of them, 140 had positive urine cultures after the first consultation. We excluded 
totally 10 women in our study, including 8 women with natural abortion or stillbirth later, 2 women defaulted follow-up after the first consultation, and 35 women with contaminated MSU culture. So 95 pregnant women were confirmed to have UTI, with the prevalence of $1.86 \%$, of whom $83(87.4 \%)$ were asymptomatic (ASB). Their mean age was $31.4 \pm 5.04$ years; range was $20-43$ years.

Table 1 and Figure 1 show the age-distribution of the 95 women with bacteriuria. ASB was commonest in the young age group. However, the occurrence of ASB had no significant association with age group, $\chi^{2}=51.23, \mathrm{p}=0.78$.

Bacteriuria occurred mostly in the multipara ( $n=57$ or $60.0 \%$, Table 2 and Figure 2). Asymptomatic bacteriuria was more often in the primipara $(n=35$ or 92.1\%). However, there was no significant association between ASB and parity; Pearson uncorrected $\chi^{2}=8.83, \mathrm{p}=0.717$.

\subsection{Bacterial Isolates}

In total, $196 \mathrm{MSU}$ specimens (cultured on first consultations and later follow-ups) showed significant bacterial isolates (Table 3). Escherichia coli (n = $111,56.6 \%$ ) was the commonest, followed by Klebsiella pneumonia ( $\mathrm{n}=20$, $10.2 \%)$, both being extended-spectrum beta-lactamases (ESBL) producers $(8.1 \%$ and $10 \%$, respectively). The next common isolates were Streptococcus agalactiae $(\mathrm{n}=16,8.2 \%)$, Klebsiella pneumonia ssp pneumonia $(\mathrm{n}=14,7.1 \%)$, and Enterobacter aerogenes $(\mathrm{n}=8.4 .1 \%)$. Of the 95 infected women, 15 women needed more than one course of antibiotics to clear the bacteriuria, and their treatment was regarded as a failure. Most bacterial isolates from the 15 women were $E$. coli $(\mathrm{n}=13,86.7 \%)$, followed by Enterobacter aerogenes and Klebsiella pneumonia.

\subsection{Antimicrobial Susceptibility}

Table 4 shows the antimicrobial susceptibility pattern of the isolated bacteria. In summary, E. coli was susceptible to nitrofurantoin $(98.2 \%)$ and the $2^{\text {nd }}$ or $3^{\text {rd }}$ generation cephalosporin (cefoxitin, ceftriaxone, ceftazidime, 88\%). K. pneumonia was susceptible to the $2^{\text {nd }}$ to $4^{\text {th }}$ generation cephalosporin (cefoxitin, cefepime, ceftriaxone, ceftazidime, 90\%), and augmentin (85.7\%). S. agalactiae was susceptible to ampicillin, penicillin Gand, nitrofurantoin (100\%, 100\%, and 75\%, respectively). Other Gram-negative bacilli, such as Klebsiella pneumonia ssp pneumoniae, were sensitive to augmentin $(80 \%)$ and the $2^{\text {nd }}$ to $4^{\text {th }}$ generation cephalosporin (>90\%).

Table 1. Distribution of bacteriuria and ASB in age groups.

\begin{tabular}{ccc}
\hline Age group (years) & $\begin{array}{c}\text { Frequency of bacteriuria } \\
\text { n (\% of total) }\end{array}$ & $\begin{array}{c}\text { Frequency of ASB in the group } \\
\text { n (\% of the group) }\end{array}$ \\
\hline $20-29$ & $36(37.9)$ & $34(94.4)$ \\
$30-39$ & $55(57.9)$ & $46(83.6)$ \\
$\geq 40$ & $4(4.2)$ & $3(75.0)$ \\
Total & $95(100)$ & $83(87.4)$ \\
\hline
\end{tabular}


Table 2. Distribution of bacteriuria and ASB in parity.

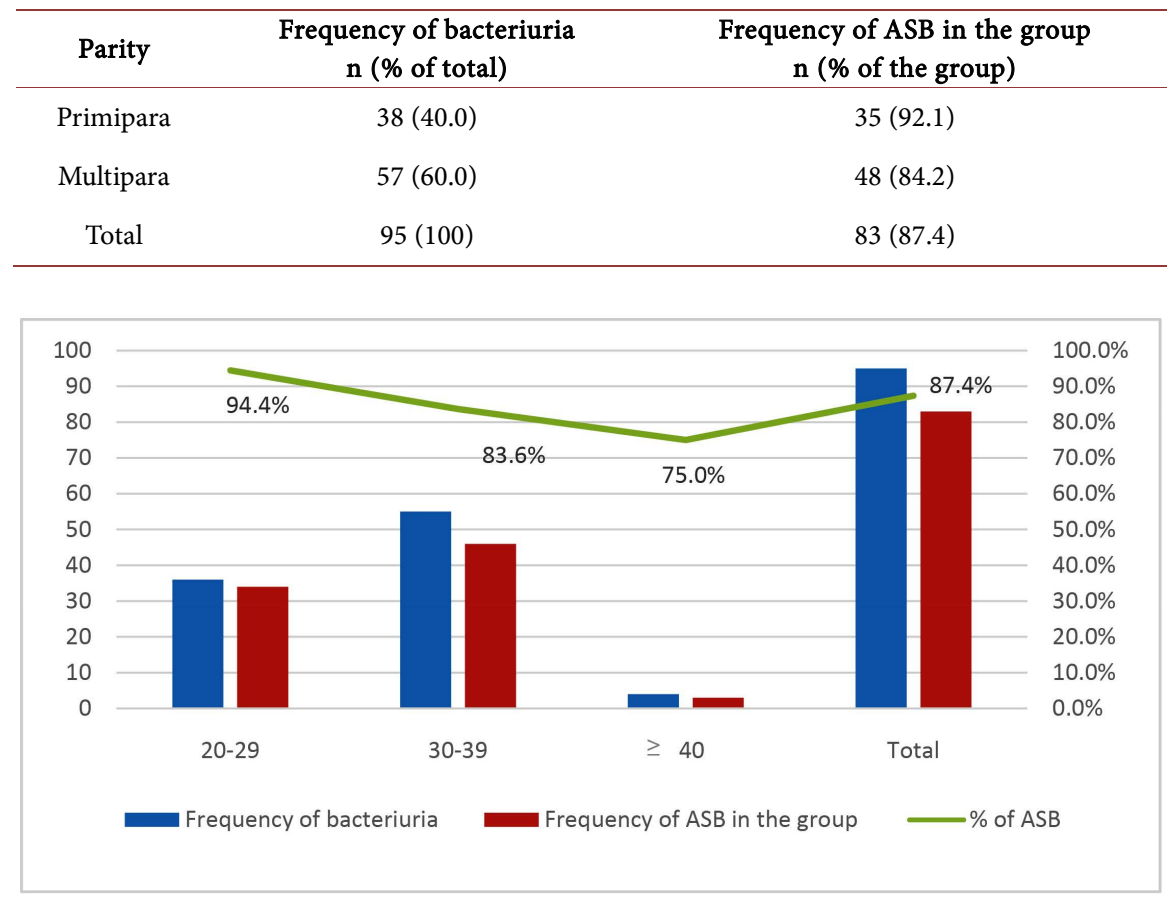

Figure 1. Distribution of bacteriuria and ASB in age groups. ASB = asymptomatic bacteriuria.

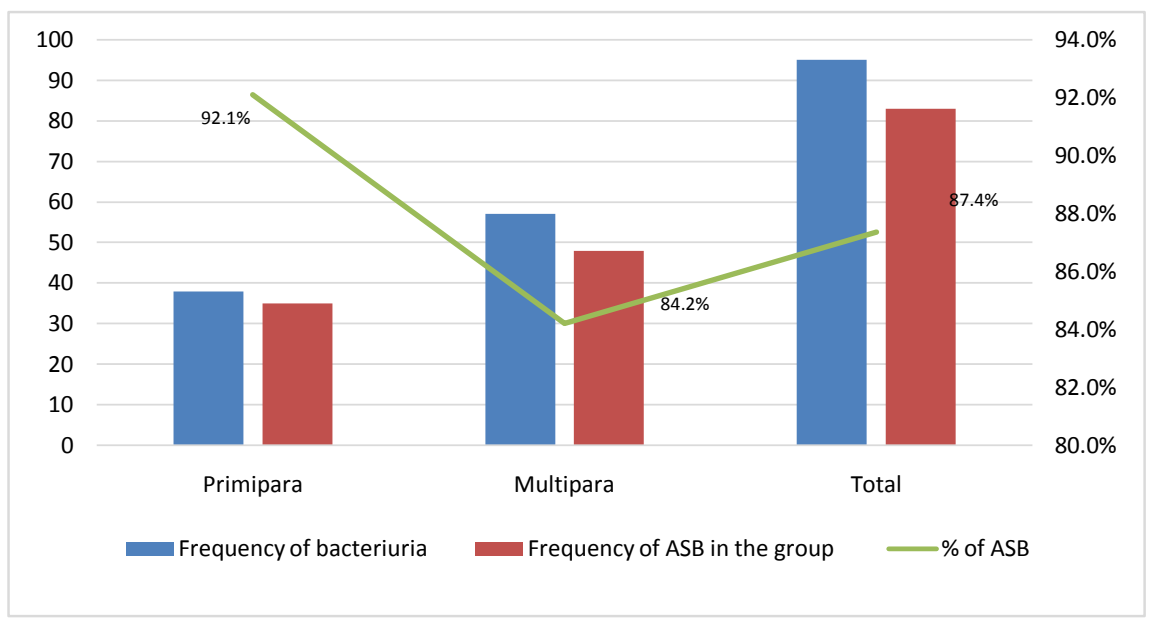

Figure 2. Distribution of bacteriuria and ASB in parity.

\subsection{Therapeutic Failure}

Of the 95 infected women, 15 (15.8\%) needed more than one course of antibiotics and their initial treatment was regarded as therapeutic failure. There were 10 bacteriuria cases to be found persistent bacteriuria through the entire trimester of pregnancy after prescribing corrected antibiotic, but those were still treated finally. 4 pregnancy women had no successful eliminate bacteriuria till delivery.

Binomial logistic regression was used to test the association between initial treatment failure and the factors collected about the pregnant women, showed as 
Table 3. Distribution of bacterial isolates in culture positive specimens.

\begin{tabular}{ccc}
\hline Types of bacterial isolate & Number of isolates $(\mathbf{n}=196)$ & Percentage \\
\hline Escherichia coli & 111 & $56.6 \%$ \\
Klebsiella pneumoniae & 20 & $10.2 \%$ \\
Streptococcus agalactiae & 16 & $8.2 \%$ \\
Klebsiella pneumonia ssp pneumoniae & 14 & $7.1 \%$ \\
Enterobacter aerogenes & 8 & $4.1 \%$ \\
Enterococcus faecalis & 7 & $3.6 \%$ \\
Candida albicans & 4 & $2.0 \%$ \\
Candida glabrata & 3 & $1.5 \%$ \\
Enterococcus spp & 3 & $1.5 \%$ \\
Others* & 10 & $5.1 \%$ \\
Total & 196 & $100 \%$ \\
\hline
\end{tabular}

${ }^{*}$ Others: Staphylococcus epidermidis (2), Staphylococcus haemolyticus (2), Enterococcus gallinarum (1), Klebsiella oxytoca (1), Staphylococcus aureus (1), Staphylococcus saprophyticus (1), Candida krusei (1), Gardnerella vaginalis (1).

Table 5. Only symptomatic UTI and the gestational age were significantly associated with therapeutic failure.

\section{Discussion}

The reported prevalence of bacteriuria varies from $2 \%$ to $13 \%$ pregnancies in affluent countries and up to $86.6 \%$ in developing and under-developed countries [9]. From this study, the prevalence of bacteriuria on the first consultation in pregnant women attending public primary care was $2.6 \%(\mathrm{n}=130)$. After the exclusion of contaminated specimens $(n=35)$, the prevalence of UTI in pregnancy is $1.9 \%(\mathrm{n}=95)$. This prevalence was higher than that in UK $(1.3 \%)$ and similar to that in US (2\%) [7] [8], but was less than those in most of the other countries, such as Brunei (4.1\%), Malaysia (4.3\%), Canada (4.7\%), Singapore (6\%) [10], Ghana (7.3\%), Iran (10\%) [11] [12], and Hong Kong (10.3\%) [13].

In this study, the highest prevalence of UTI was found in the middle-age group of 30 - 39 years $(57.9 \%)$ and least in the group over 39 years (4.2\%). This may be relative to the childbearing age was mainly in middle-aged, and the median age of the pregnant women in our study was $31.4 \pm 5.04$ years. This was similar to the findings by Turpin et al. [14], the highest rate of $53 \%$ being in the age-group of 35 - 39 years. Furthermore, the prevalence of pregnancy UTI increases with early trimesters and in women of multiparity (60.0\%)-similar to the findings by Sujatha R et al. and Roy et al. [15] [16].

Primary health care in Macau is very concerned about primary prevention of maternal diseases, especially for those who are treatable and have serious consequences, such as UTI. In Macau, routine UTI screening program in antenatal care had been carried out in all government health centers for nearly 5 years. 
Table 4. Antimicrobial susceptibility pattern of isolated organisms.

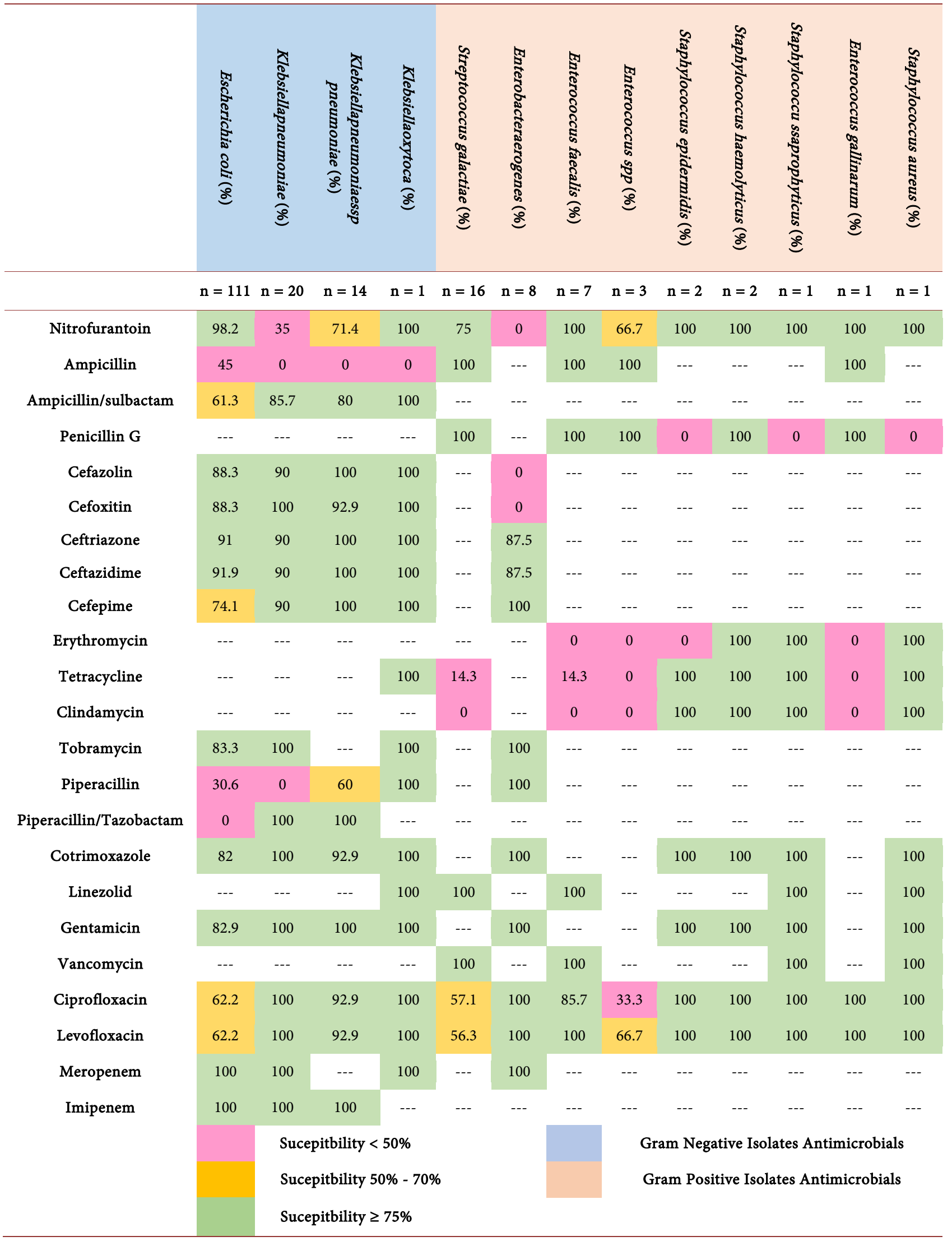


Table 5. Risk factors among therapeutic failure and characteristics of the pregnant women, the logistic regression model.

\begin{tabular}{cccccc}
\hline Characteristics & $\begin{array}{c}\text { Therapeutic } \\
\text { success, } \\
\text { frequency }(\%)^{*}\end{array}$ & $\begin{array}{c}\text { Therapeutic } \\
\text { failure, } \\
\text { frequency }(\%)^{*}\end{array}$ & B & $95 \%$ CI & $P$ \\
\hline Average age & $31.4 \pm 5.05$ & $31.1 \pm 0.15$ & 0.72 & $-0.98,2.52$ & 0.41 \\
\hline Parity & & & & \\
\hline Primipara & $31(38.8)$ & $7(46.7)$ & -1.62 & $-0.98,2.52$ & 0.25 \\
Multipara & $49(61.3)$ & $8(53.3)$ & 0.73 & $-0.86,2.48$ & 0.38 \\
\hline Gestational age & & & & \\
\hline $4-11^{+6} \mathrm{w}$ & $70(87.5)$ & $10(66.7)$ & 0.92 & $-2.66,2.55$ & 0.76 \\
$12-27^{+6} \mathrm{w}$ & $5(6.3)$ & $1(6.7)$ & -0.58 & $-3.18,2.71$ & 0.68 \\
$\geq 28 \mathrm{w}$ & $5(6.3)$ & $4(26.7)$ & -2.41 & $-4.42,-0.49$ & $0.01^{\dagger}$ \\
\hline Others & & & & \\
\hline Anemia & $36(45)$ & $4(26.7)$ & 1.32 & $-0.26,3.24$ & 0.13 \\
GDM & $10(12.5)$ & $3(20.0)$ & -0.67 & $-2.29,1.12$ & 0.43 \\
History of UTI & $6(7.5)$ & $4(26.7)$ & -0.50 & $-2.51,1.70$ & 0.63 \\
Symptomatic & $6(7.5)$ & $6(40)$ & -2.74 & $-4.81,-0.96$ & $\mathbf{0 . 0 0 4}{ }^{\dagger}$ \\
\hline
\end{tabular}

According to the antimicrobial drug susceptibility testing: ${ }^{\star}$ Percentage (\%) is taken within the therapeutic group. $\uparrow p$-value of $\leq 0.05$ was considered statistically significant.

Our pregnant women in Macau health centre have a relatively low prevalence of UTI, Screening UTI for the implementation of program seems to be effective. High proportion of asymptomatic bacteriuria (87.4\%) in pregnancy women was found in our study; ASB occurs more often among the young and the primipara women who are less experienced with pregnancy. Strengthening prenatal education and continuing to screen UTI is still warranted due to the potential hazards of UTI complications. It also can help improve the quality of antenatal care subsequently and is an important part of primary health care.

\subsection{Microbiology}

Of the 196 positive cultures, the Gram-negative bacteria were more prevalent ( $\mathrm{n}$ $=146,74.5 \%)$ than the Gram-positive ones $(n=41,20.9 \%)$ and Candida $(n=9$, $4.6 \%)$. The common bacteria isolated were similar to those observed in Turkey [17], South Africa [18], and Hong Kong [13].

The bacteria frequently isolated in this study were highly sensitive to the common first-line antibiotics like nitrofurantoin. E.g., E. coli was $98.2 \%$ sensitive to nitrofurantoin, $K$. pneumonia $85.7 \%$ sensitive to augmentin, and $S$. agalactiae $100 \%$ sensitive to ampicillin and $75 \%$ to nitrofurantoin. This high susceptibility to commonly used antibiotics may well be the result of the strict antibiotic supervision by the Macau health authority. (Antibiotic consumption is supervised by the Office of Pharmaceutical Affairs, Antibiotic Management Committee in Central Hospital, and the infection control team of Health Centre every quarter of the year). 
This study showed a high rate $(26.9 \%)$ of contaminated specimens collected from the asymptomatic women following the first antenatal consultation. This finding highlights the importance of educating the correct method of urine sample collection, and repeating the MSU culture in ASB before prescribing an antibiotic, to avoid unnecessary treatment and antibiotic abuse.

\subsection{Therapy}

There is no clear consensus in the literature on antibiotic choice or duration of treatment for urinary tract infection. In this study, 15 women received more than one course of antibiotics. The bacterial isolates in their MSU cultures were predominately E. coli (86.7\%); they were similar to the bacterial isolates of the therapeutic successful group. From the logistic regression, such failure cases were significantly associated with symptomatic UTI and later trimester, but not with other factors considered in this study. The risk factors can easily be identified. But the mechanism of therapeutic failure has been scarcely studied. Given that the bacterial isolates and the prevalence of diabetes mellitus were similar between the success and failure groups, other pathophysiological mechanisms should further be studied, e.g., treatment compliance, optimal dosage and/or duration of antibiotics, and other co-morbid conditions were not included in this study.

\section{Limitation}

This study did not investigate the risk factors of bacteriuria. The outcome of patients with bacteriuria was also not assessed, for which further studies are necessary. Furthermore, further urine screening in later trimesters was not assessed while some studies showed improved outcomes by such screening. Information from this study is limited to the initial assessment of pregnant women attending primary care for antenatal care.

\section{Conclusion}

The prevalence of bacteriuria in pregnant women attending public primary care in Macau for antenatal care was $1.86 \%$, of which $87.4 \%$ were ASB. ASB was mainly found in younger ages from 20 to 30 years and the primipara. E. coli, $K$. pneumonia, and $S$. agalactiae were the commonest pathogens. In the urine specimens from the first collection, $26.9 \%$ were contaminated. Initial antimicrobial treatment was not effective (therapeutic failure) in $15.8 \%$. Symptomatic UTI and late trimester were significantly associated with therapeutic failure.

\section{Acknowledgements}

Centro Hospitalar Conde de São Januário, Department of Family Medicine, Department of Clinical Pathology, Computer Department, Areia Preta Health Center, Alameda Dr. Carlos d'Assumpcao, Macau, China; thank you also to Prof. Yuk-Tsan Wun for professional assistance in modifying manuscript. 


\section{Conflicts of Interest}

The authors declare no conflicts of interest regarding the publication of this paper.

\section{References}

[1] Patterson, T.F. and Andriole, V.T. (1997) Detection, Significance, and Therapy of Bacteriuria in Pregnancy. Update in the Managed Health Care Era. Infectious Disease Clinics of North America, 11, 593-608. https://doi.org/10.1016/S0891-5520(05)70375-5

[2] Romero, R., Oyarzun, E., Mazor, M., et al. (1989) Meta-Analysis of the Relationship between Asymptomatic Bacteriuria and Preterm Delivery/Low Birth Weight. $O b$ stetrics \& Gynecology, 73, 576-582.

[3] Nicolle, L.E., Bradley, S., Colgan, R., et al. (2005) Infectious Diseases Society of America Guidelines for the Diagnosis and Treatment of Asymptomatic Bacteriuria in Adults. Clinical Infectious Diseases, 40, 643-654. https://doi.org/10.1086/427507

[4] Minassian, C., Thomas, S.L., Williams, D.J., et al. (2013) Acute Maternal Infection and Risk of Pre-Eclampsia: A Population-Based Case-Control Study. PLoS ONE, 8, e73047. https://doi.org/10.1371/journal.pone.0073047

[5] Hooton, T.M. and Gupta, K. (2019) Urinary Tract Infections and Asymptomatic Bacteriuria in Pregnancy. Uptodata.

[6] Wingert, A. and Pillay, J. (2019) Asymptomatic Bacteriuria in Pregnancy: Systematic Reviews of Screening and Treatment Effectiveness and Patient Preferences. BMJ Open, 9, e021347. https://doi.org/10.1136/bmjopen-2017-021347

[7] Johnson, E.K., et al. (2018) Urinary Tract Infections in Pregnancy. O \& G Medscape.

[8] Smaill, F.M. and Vazquez, J.C. (2015) Antibiotics for Asymptomatic Bacteriuria in Pregnancy. Cochrane Database of Systematic Reviews, CD000490. https://doi.org/10.1002/14651858.CD000490.pub3

[9] Alemu, A., Mogus, F., Shiferaw, Y., Tefas, A., Kassu, A., Anagaw, B., et al. (2012) Bacterial Profiles and Drug Susceptibility Patterns of Urinary Tract Infection in Pregnant Women at University of Gonda Teaching Hospital North West Ethiopia. BMC Research Notes, 5, Article No. 197. https://doi.org/10.1186/1756-0500-5-197

[10] McCormick, T., Ashe, R.G. and Kearney, P.M. (2008) Review Urinary Tract Infection in Pregnancy. The Obstetrician \& Gynaecologist, 10, 156-162. https://doi.org/10.1576/toag.10.3.156.27418

[11] Nilekar, S.L., et al. (2015) Clinical Bacteriological Study of Urinary Tract Infection in Pregnant Women. IOSR Journal of Dental and Medical Sciences, 14, 43-49.

[12] Akerele, P.F. and Okonofua, A. (2001) Prevalence of Asymptomatic Bacteriuria among Pregnant Women in Benin City, Nigeria. Journal of Obstetrics and Gynaecology, 21, 141-144. https://doi.org/10.1080/01443610020026038

[13] Fong, S.Y., Tung, C.W. and Yu, F.N.Y. (2013) The Prevalence of Asymptomatic Bacteriuria in Pregnant Hong Kong Women. Hong Kong Journal of Gynaecology, Obstetrics and Midwifery, 13, 43.

[14] Turpin, C., Minkah, B., Danso, K., et al. (2007) Asymptomatic Bacteriuria in Pregnant Women Attending Antenatal Clinic at Komfo Anokye Teaching Hospital, Kumasi, Ghana. Ghana Medical Journal, 41, 26-29.

[15] Sujatha, R. and Nawan, M. (2014) Prevalence of Asymptomatic Bacteriuria and Its 
Antibacterial Susceptibility Pattern among Pregnant Women Attending the Antenatal Clinic at Kanpur, India. Journal of Clinical and Diagnostic Research, 8, DC01DC03. https://doi.org/10.7860/JCDR/2014/6599.4205

[16] Pais, P., Khurana, R. and George, J. (2002) Urinary Tract Infections: A Retrospective Survey of Causative Organisms and Antibiotics Prescribed in a Tertiary Care Setting. Indian Journal of Pharmacology, 34, 278-280.

[17] Gilbert, N.M., O'Brien, V.P. and Hultgren, S. (2013) Urinary Tract Infection as a Preventable Cause of Pregnancy. Complications: Opportunities, Challenges, and a Global Call to Action. Global Advances in Health and Medicine, 2, 59-69. https://doi.org/10.7453/gahmj.2013.061

[18] Jain, V., Das, V., Agarwal, A. and Pandey, A. (2013) Asymptomatic Bacteriuria and Obstetric Outcome Following Treatment in Early Versus Late Pregnancy in North Indian Women. Indian Journal of Medical Research, 137, 753-758.

\section{Abbreviation}

ASB: Asymptomatic Bacteriuria

SB: Symptomatic Bacteriuria

UTI: Urinary Tract Infection

MSU: Mid-Stream Urine

CFU: Colony-Forming Unit

CHCSJ: Centro Hospital ar Conde de São Januário

GDM: Gestational Diabetes Mellitus

ESBL: Extended-Spectrum Beta-Lactamases 